JINR-E2-94-400

October 1994

\title{
RENORMALIZATION GROUP IMPROVED RADIATIVE CORRECTIONS TO THE SUPERSYMMETRIC HIGGS BOSON MASSES
}

\author{
A.V.Gladyshev' and D.I.Kazakov? \\ Bogoliubov Laboratory of Theoretical Physics, Joint Institute for Nuclear Research, \\ 141980 Dubna, Moscow Region, RUSSIA
}

\begin{abstract}
The one-loop radiative corrections to the Higgs boson potential in the MSSM, originating from the top quark and squark loops, are summed in the leading log approximation using the renormalization group. The RG improved effective potential is minimized and the corrections to the CP-odd and CP-even Higgs boson masses are calculated. The resulting masses exhibit smoother top mass dependence than those calculated without RG summation. We have also found that for preferable values of the top mass the light Higgs mass does not exceed $100 \mathrm{GeV}$.
\end{abstract}

\footnotetext{
${ }^{1}$ Physics Department, Moscow State University; e-mail: gladysh@thsun1.jinr.dubna.su

${ }^{2}$ Supported in part by INS Grant \#RFL000; e-mail: kazakovd@thsun1.jinr.dubna.su
} 


\section{Introduction}

It has been recently pointed out [1, 2] that the radiative corrections to the masses of the Higgs bosons in the framework of the MSSM [3], [4], [5] can be relatively large. The leading correction to the effective potential comes from the top quark and squark loops, being proportional to the top Yukawa coupling, which is considered to be big due to the heaviness of the top quark. The other corrections happen to be smaller, though in some cases their effect is not negligible as well. The net effect of the radiative corrections is to increase the masses of the Higgs bosons. This increase may be very significant for the future searches, since it can achieve several dozen $\mathrm{GeV}$, implying that the Higgs mass could exceed the $Z$ boson mass.

Considering the tree-level Higgs potential one finds out that the value of the lightest Higgs boson mass is restricted by the inequality

$$
m_{h}<M_{Z}
$$

This strict limit is, however, violated by the radiative corrections. The radiative corrections to the supersymmetric boson masses proceed ifrom the one-loop effective potential

$$
\begin{aligned}
V & =V_{\text {tree }}+\Delta V_{1 \text { loop }}, \\
\Delta V_{\text {lloop }} & =\frac{1}{64 \pi^{2}} \operatorname{Str}_{\mathcal{M}}^{4}\left(\log \frac{\mathcal{M}^{2}}{Q^{2}}-\frac{3}{2}\right),
\end{aligned}
$$

where $\operatorname{Str}$ denotes the conventional supertrace and $Q^{2}$ is the scale at which all the couplings in the tree-level potential are renormalized. $\mathcal{M}$ are the field dependent masses of all the possible particles running through the loops. In what follows we limit ourselves with the top and stop contributions as the main ones.

As far as the radiative corrections appear to be large achieving $30 \%$ one can wonder about the values of the higher order contributions. Since according to eq.(3) they have the $\log$ form one is expecting to have $\log ^{2}$ at the second loop, $\log ^{3}$ at the third loop, etc. Being essential these logs have to be summed giving considerable change of the results.

Indeed this happens in the simplest case of the $\phi^{4}$ model considered in the pioneering paper by Coleman and Weinberg [6]. The summation of the leading logs to the effective potential changed the situation qualitatively leading to the disappearance of a non-trivial minimum arising at the one-loop level.

The summation procedure can be naturally done with the help of the renormalization group technique, which we are going to apply to our particular case. 


\section{RG Improved Effective Potential}

Let us remind the expression for the one-loop effective Higgs potential in the MSSM which takes into account the radiative corrections due to the top quark and squark loops. For the neutral Higgses it has been calculated in ref. [1] and has the form

$$
\begin{gathered}
V\left(H_{1}, H_{2}\right)=m_{1}^{2}\left|H_{1}\right|^{2}+m_{2}^{2}\left|H_{2}\right|^{2}-m_{3}^{2}\left(H_{1} H_{2}+\text { h.c. }\right)+\frac{g^{2}+g^{\prime 2}}{8}\left(\left|H_{1}\right|^{2}-\left|H_{2}\right|^{2}\right)^{2} \\
+\frac{3}{32 \pi^{2}}\left[\tilde{m}_{t 1}^{4}\left(\ln \frac{\tilde{m}_{t 1}^{2}}{Q^{2}}-\frac{3}{2}\right)+\tilde{m}_{t 2}^{4}\left(\ln \frac{\tilde{m}_{t 2}^{2}}{Q^{2}}-\frac{3}{2}\right)-2 m_{t}^{4}\left(\ln \frac{m_{t}^{2}}{Q^{2}}-\frac{3}{2}\right)\right]-V_{0},
\end{gathered}
$$

where $\tilde{m}_{t i}$ are the field dependent masses of the stop particles and $m_{t}$ is the field dependent top mass. The scale $Q^{2}$ remains arbitrary and is usually chosen to be equal to the value of the top mass. In fact the potential is scale independent since explicit dependence on the scale is compensated by the implicit dependence of the parameters renormalized at this scale. $V_{0}$ is the value of the potential at $H_{1}=H_{2}=0$, which has to be subtracted in order to keep the scale invariance [8].

The field dependent squarks masses are given by the eigenvalues of the mass-squared matrix

$$
\left(\begin{array}{cc}
m_{Q}^{2}+h_{t}^{2}\left|H_{2}^{0}\right|^{2}+\left(g^{2}-\frac{1}{3} g^{2}\right)\left(\left|H_{1}^{0}\right|^{2}-\left|H_{2}^{0}\right|^{2}\right) / 4 & h_{t}\left(A_{t} H_{2}^{0}+\mu H_{1}^{0 *}\right) \\
h_{t}\left(A_{t} H_{2}^{0 *}+\mu H_{1}^{0}\right) & m_{U}^{2}+h_{t}^{2}\left|H_{2}^{0}\right|^{2}+\frac{1}{3} g^{\prime 2}\left(\left|H_{1}^{0}\right|^{2}-\left|H_{2}^{0}\right|^{2}\right)
\end{array}\right)
$$

where $A_{t}$ is the conventional trilinear soft supersymmetry breaking parameter, $\mu$ is the Higgs mixing parameter, and the top mass squared is given by $h_{t}^{2}\left|H_{2}^{0}\right|^{2}$. The so-called Dterms give contribution proportional to the gauge couplings and will be ignored hereafter in order to gain approximate scale independence of the potential, since we are including only top-stop contributions to $\Delta V$.

Then the eigenvalues of the matrix (5) are

$$
\tilde{m}_{t_{1,2}}^{2}=m_{t}^{2}+\frac{1}{2}\left[m_{Q}^{2}+m_{U}^{2} \pm \sqrt{\left(m_{Q}^{2}-m_{U}^{2}\right)^{2}+4 m_{t}^{2}\left(A_{t}+\mu \cot \beta\right)^{2}}\right]
$$

where $\tan \beta=\left|H_{2}^{0}\right| /\left|H_{1}^{0}\right|$.

The scale independence of the effective potential (幽) is given by the renormalization group equation

$$
Q^{2} \frac{d}{d Q^{2}} V=\left(Q^{2} \frac{\partial}{\partial Q^{2}}+\beta_{m_{i}} \frac{\partial}{\partial m_{i}^{2}}+\gamma_{1} H_{1} \frac{\partial}{\partial H_{1}}+\gamma_{2} H_{2} \frac{\partial}{\partial H_{2}}\right) V=0
$$


where $m_{i}$ are the parameters of the tree-level potential and $\beta$ 's denote their $\beta$ functions. According to the approximation mentioned above, we have ignored the scale dependence of the gauge couplings.

The general solution of eq.(7) has the form:

$$
\hat{V}=V\left(m_{i}(t), H_{i}(t), t=0\right)
$$

where the function $V(m, H, t)$ is the perturbative expression with the scale chosen arbitrary, $m_{i}$ - are the effective parameters of the potential,

$$
H_{i}(t)=H_{i} \xi_{i}^{1 / 2}(t), \quad \xi_{i}=\exp \int_{0}^{t} \gamma_{i}\left(t^{\prime}\right) d t^{\prime}, \quad t=\ln \frac{M_{X}^{2}}{Q^{2}}
$$

and $\gamma^{\prime}$ 's are anomalous dimensions of the fields $H_{i}$. In particular one can choose $Q^{2}$ to be equal to the top mass, as is usually done. However, the main distinction is whether we choose the top mass to be field dependent and put it equal to its numerical value after the minimization of the potential, or we take its numerical value at the very beginning and then minimize the potential. In the latter case we incorporate the perturbative corrections to the potential, while in the first case we sum all the leading logs via the renormalization group equation. Proceeding the first way we get

$$
\begin{aligned}
V\left(H_{1}, H_{2}\right) & =m_{1}^{2}\left(m_{t}\right)\left|H_{1}\right|^{2}+m_{2}^{2}\left(m_{t}\right)\left|H_{2}\right|^{2}-m_{3}^{2}\left(m_{t}\right)\left(H_{1} H_{2}+\text { h.c. }\right) \\
& +\frac{g^{2}+g^{2}}{8}\left(\left|H_{1}\right|^{2}-\left|H_{2}\right|^{2}\right)^{2} \\
& +\frac{3}{32 \pi^{2}}\left[\tilde{m}_{t 1}^{4}\left(\ln \frac{\tilde{m}_{t 1}^{2}}{m_{t}^{2}}-\frac{3}{2}\right)+\tilde{m}_{t 2}^{4}\left(\ln \frac{\tilde{m}_{t 2}^{2}}{m_{t}^{2}}-\frac{3}{2}\right)+3 m_{t}^{4}\right. \\
& \left.-\tilde{m}_{Q}^{4}\left(\ln \frac{\tilde{m}_{Q}^{2}}{m_{t}^{2}}-\frac{3}{2}\right)+\tilde{m}_{D}^{4}\left(\ln \frac{\tilde{m}_{Q}^{2}}{m_{t}^{2}}-\frac{3}{2}\right)\right],
\end{aligned}
$$

where $m_{t}$ is the field dependent mass and $m_{Q}$ and $m_{D}$ are the stop masses boundary values when $H_{1}=H_{2}=0$.

Eq.8 is the RG improved expression for the one-loop effective potential which corresponds to taking into account all the leading log contributions proportional to the top Yukawa coupling from all the loops. 


\section{Minimization of the Potential}

To find the vacuum expectation values of the Higgs fields we have to minimize the potential. Using the notation

$$
\left\langle H_{1}^{0} \xi_{1}^{1 / 2}\right\rangle=v_{1},\left\langle H_{2}^{0} \xi_{2}^{1 / 2}\right\rangle=v_{2}, v^{2}=v_{1}^{2}+v_{2}^{2}, \tan \beta=v_{2} / v_{1}
$$

and keeping only the terms of the first order in coupling constants the minimum of the potential (8) is given by

$$
\begin{aligned}
v^{2} & =\frac{4}{\left(g^{2}+g^{2}\right)\left(\tan ^{2} \beta-1\right)\left(1+\varepsilon_{1}\right)}\left\{m_{1}^{2}\left(1+\gamma_{1}\right)-m_{2}^{2}\left(1-\gamma_{1}\right) \tan ^{2} \beta+\varepsilon_{2} \tan ^{2} \beta\right. \\
& \left.\left.-\frac{3 h_{t}^{2}}{16 \pi^{2}}\left[f\left(\tilde{m}_{t 1}^{2}\right)+f\left(\tilde{m}_{t 2}^{2}\right)+2 m_{t}^{2}\right] \tan ^{2} \beta+\left(A_{t}^{2} \tan ^{2} \beta-\mu^{2}\right) \frac{\left.f\left(\tilde{m}_{t 1}^{2}\right)-f\left(\tilde{m}_{t 2}^{2}\right)\right]}{\tilde{m}_{t 1}^{2}-\tilde{m}_{t 2}^{2}}\right]\right\} \\
2 m_{3}^{2} & =\frac{\sin 2 \beta}{1+\varepsilon_{1}}\left\{m_{1}^{2}\left(1-\gamma_{2}\right)+m_{2}^{2}\left(1-\gamma_{1}\right)-\varepsilon_{2}\right. \\
& \left.+\frac{3 h_{t}^{2}}{16 \pi^{2}}\left[f\left(\tilde{m}_{t 1}^{2}\right)+f\left(\tilde{m}_{t 2}^{2}\right)-2 f\left(m_{t}^{2}\right)+\left(A_{t}+\mu \tan \beta\right)\left(A_{t}+\mu \cot \beta\right) \frac{f\left(\tilde{m}_{t 1}^{2}\right)-f\left(\tilde{m}_{t 2}^{2}\right)}{\tilde{m}_{t 1}^{2}-\tilde{m}_{t 2}^{2}}\right]\right\}
\end{aligned}
$$

where

$$
\begin{aligned}
f\left(m^{2}\right) & =m^{2}\left(\log \frac{m^{2}}{m_{t}^{2}}-1\right) \\
\varepsilon_{1} & =\gamma_{1} \cos ^{2} \beta-\gamma_{2} \sin ^{2} \beta \\
\varepsilon_{2} & =3\left[\left(\tilde{\alpha}_{2} M_{2}^{2}+\frac{1}{5} \tilde{\alpha}_{1} M_{1}^{2}+\tilde{\alpha}_{2} \mu^{2}+\frac{1}{5} \tilde{\alpha}_{1} \mu^{2}\right)\left(\cot ^{2} \beta+1\right)+2\left(\tilde{\alpha}_{2} M_{2}+\frac{1}{5} \tilde{\alpha}_{1} M_{1}\right) \mu \cot \beta\right], \\
\gamma_{1} & =-\frac{3}{2}\left(\tilde{\alpha}_{2}+\frac{1}{5} \tilde{\alpha}_{1}\right) \\
\gamma_{2} & =\frac{3}{2}\left(2 Y_{t}-\tilde{\alpha}_{2}-\frac{1}{5} \tilde{\alpha}_{1}\right) \\
Y_{t} & =\frac{h_{t}^{2}}{16 \pi^{2}}, \tilde{\alpha}_{i}=\frac{\alpha_{i}}{4 \pi}=\frac{g_{i}^{2}}{16 \pi^{2}} .
\end{aligned}
$$

Here the values of all the mass parameters and couplings are taken at the scale equal to the top mass. 


\section{Corrections to the Higgs Masses}

Having in mind eqs.(10) we are now in a position to calculate the RG improved radiative corrections to the masses.

One has:

$$
M_{Z}^{2}=2 \frac{m_{1}^{2}\left(1+\gamma_{1}\right)-m_{2}^{2}\left(1-\gamma_{1}\right) \tan ^{2} \beta-\tilde{\Delta}_{Z}^{2}}{\left(\tan ^{2} \beta-1\right)\left(1+\varepsilon_{1}\right)}
$$

where

$$
\tilde{\Delta}_{Z}^{2}=\Delta_{Z}^{2}-\varepsilon_{2} \tan ^{2} \beta
$$

and $\Delta_{Z}^{2}$ is the one-loop radiative correction [1], [7]

$$
\Delta_{Z}^{2}=\frac{3 g^{2}}{32 \pi^{2}} \frac{m_{t}^{2}}{M_{W}^{2} \cos ^{2} \beta}\left[f\left(\tilde{m}_{t 1}^{2}\right)+f\left(\tilde{m}_{t 2}^{2}\right)+2 m_{t}^{2}+\left(A_{t}^{2} m_{0}^{2}-\mu^{2} \cot ^{2} \beta\right) \frac{f\left(\tilde{m}_{t 1}^{2}\right)-f\left(\tilde{m}_{t 2}^{2}\right)}{\tilde{m}_{t 1}^{2}-\tilde{m}_{t 2}^{2}}\right]
$$

Using eqs.(11) we can also calculate the corrections to the squared masses in the CPodd neutral sector. Just like in the usual case [1] taking the second derivative of the full potential with respect to $\phi_{i} \equiv \operatorname{Im} H_{i}^{0}$ one has:

$$
\left.\left(\frac{\partial^{2} V}{\partial \phi_{i} \partial \phi_{j}}\right)\right|_{v_{1}, v_{2}}=\left(\begin{array}{cc}
\tan \beta & 1 \\
1 & \cot \beta
\end{array}\right) \Delta
$$

where

$$
\Delta=2 m_{3}^{2}-\frac{3 g^{2}}{32 \pi^{2} \sin ^{2} \beta} \frac{m_{t}^{2}}{M_{W}^{2}} A m_{0} \mu \frac{f\left(\tilde{m}_{t 1}^{2}\right)-f\left(\tilde{m}_{t 2}^{2}\right)}{\tilde{m}_{t 1}^{2}-\tilde{m}_{t 2}^{2}}
$$

This gives for the CP-odd Higgs mass

$$
m_{A}^{2}=\frac{1}{1+\varepsilon_{1}}\left\{m_{1}^{2}\left(1-\gamma_{2}\right)+m_{2}^{2}\left(1-\gamma_{1}\right)+\tilde{\Delta}_{A}^{2}\right\}
$$

where

$$
\tilde{\Delta}_{A}^{2}=\Delta_{A}^{2}-\varepsilon_{2}
$$

and

$$
\Delta_{A}^{2}=\frac{3 g^{2}}{32 \pi^{2}} \frac{m_{t}^{2}}{M_{W}^{2} \sin ^{2} \beta}\left[f\left(\tilde{m}_{t 1}^{2}\right)+f\left(\tilde{m}_{t 2}^{2}\right)+2 m_{t}^{2}+\left(A_{t}^{2} m_{0}^{2}+\mu^{2}\right) \frac{f\left(\tilde{m}_{t 1}^{2}\right)-f\left(\tilde{m}_{t 2}^{2}\right)}{\tilde{m}_{t 1}^{2}-\tilde{m}_{t 2}^{2}}\right]
$$

For the CP-even sector we have to differentiate the potential with respect to $\psi_{i}=$ $R e H_{i}^{0}$. We find 


$$
\begin{gathered}
\left.\left(\frac{\partial^{2} V}{\partial \psi_{i} \partial \psi_{j}}\right)\right|_{v_{1}, v_{2}}=\left(\begin{array}{cc}
\cot \beta & -1-\delta_{1} \\
-1-\delta_{1} & \tan \beta\left(1+\delta_{2}\right)
\end{array}\right) M_{Z}^{2} \sin 2 \beta \\
+\left(\begin{array}{cc}
\tan \beta & -1-\delta_{3} \\
-1-\delta_{3} & \cot \beta\left(1+\delta_{4}\right)
\end{array}\right) \Delta+2\left(\begin{array}{cc}
\Delta_{11} & \Delta_{12} \\
\Delta_{12} & \Delta_{22}
\end{array}\right)+2\left(\begin{array}{cc}
0 & -\delta_{5} \\
-\delta_{5} & \delta_{6}
\end{array}\right),
\end{gathered}
$$

where

$$
\begin{aligned}
\delta_{1}= & \gamma_{1} \cot ^{2} \beta-\gamma_{2}, \\
\delta_{2}= & \left(\gamma_{1}+\gamma_{2}\right)\left(\cot ^{2} \beta-1\right), \\
\delta_{3}= & 2 \gamma_{1}, \\
\delta_{4}= & 2 \gamma_{2}, \\
\delta_{5}= & 6\left[\left(\tilde{\alpha}_{2} M_{2}^{2}+\frac{1}{5} \tilde{\alpha}_{1} M_{1}^{2}+\tilde{\alpha}_{2} \mu^{2}+\frac{1}{5} \tilde{\alpha}_{1} \mu^{2}\right) \cot ^{2} \beta+2\left(\tilde{\alpha}_{2} M_{2}+\frac{1}{5} \tilde{\alpha}_{1} M_{1}\right) \mu\right], \\
\delta_{6}= & 6\left[\left(\tilde{\alpha}_{2} M_{2}^{2}+\frac{1}{5} \tilde{\alpha}_{1} M_{1}^{2}+\tilde{\alpha}_{2} \mu^{2}+\frac{1}{5} \tilde{\alpha}_{1} \mu^{2}\right)\left(\cot ^{2} \beta-1\right)+2\left(\tilde{\alpha}_{2} M_{2}+\frac{1}{5} \tilde{\alpha}_{1} M_{1}\right) \mu \cot \beta\right. \\
& \left.-A m_{0} \mu Y \cot \beta\right]
\end{aligned}
$$

and $\Delta_{i j}$ 's are [1]:

$$
\begin{aligned}
\Delta_{11}= & \frac{3 g^{2}}{16 \pi^{2}} \frac{m_{t}^{4}}{\sin ^{2} \beta M_{W}^{2}}\left[\frac{\mu\left(A_{t} m_{0}+\mu \cot \beta\right)}{\tilde{m}_{t 1}^{2}-\tilde{m}_{t 2}^{2}}\right]^{2} d\left(\tilde{m}_{t 1}^{2}, \tilde{m}_{t 2}^{2}\right), \\
\Delta_{22}= & \frac{3 g^{2}}{16 \pi^{2}} \frac{m_{t}^{4}}{\sin ^{2} \beta M_{W}^{2}}\left[\ln \left(\frac{\tilde{m}_{t 1}^{2} \tilde{m}_{t 2}^{2}}{m_{t}^{4}}\right)+\frac{2 A_{t} m_{0}\left(A_{t} m_{0}+\mu \cot \beta\right)}{\tilde{m}_{t 1}^{2}-\tilde{m}_{t 2}^{2}} \ln \left(\frac{\tilde{m}_{t 1}^{2}}{\tilde{m}_{t 2}^{2}}\right)\right. \\
& \left.+\left[\frac{A_{t} m_{0}\left(A_{t} m_{0}+\mu \cot \beta\right)}{\tilde{m}_{t 1}^{2}-\tilde{m}_{t 2}^{2}}\right]^{2} d\left(\tilde{m}_{t 1}^{2}, \tilde{m}_{t 2}^{2}\right)\right], \\
\Delta_{12}= & \frac{3 g^{2}}{16 \pi^{2}} \frac{m_{t}^{4}}{\sin ^{2} \beta M_{W}^{2}} \frac{\mu\left(A_{t} m_{0}+\mu \cot \beta\right)}{\tilde{m}_{t 1}^{2}-\tilde{m}_{t 2}^{2}}\left[\ln \left(\frac{\tilde{m}_{t 1}^{2}}{\tilde{m}_{t 2}^{2}}\right)+\frac{A_{t} m_{0}\left(A_{t} m_{0}+\mu \cot \beta\right)}{\tilde{m}_{t 1}^{2}-\tilde{m}_{t 2}^{2}} d\left(\tilde{m}_{t 1}^{2}, \tilde{m}_{t 2}^{2}\right)\right] \\
& h\left(m^{2}\right)=\frac{m^{2}}{m^{2}-\tilde{m}_{q}^{2}} \ln \frac{m^{2}}{\tilde{m}_{q}^{2}} \quad d\left(m_{1}^{2}, m_{2}^{2}\right)=2-\frac{m_{1}^{2}+m_{2}^{2}}{m_{1}^{2}-m_{2}^{2}} \ln \frac{m_{1}^{2}}{m_{2}^{2}},
\end{aligned}
$$

$\tilde{m}_{q}^{2}$ is the mass of a light squark.

The diagonalization of the matrix (16) gives us the masses of the CP-even neutral Higgses.

For the charged ones one has the usual expression [1]

$$
m_{H^{ \pm}}^{2}=m_{A}^{2}+M_{W}^{2}+\Delta_{H}^{2}
$$


where

$$
\Delta_{H}^{2}=-\frac{3 g^{2}}{32 \pi^{2}} \frac{m_{t}^{4} \mu^{2}}{\sin ^{4} \beta M_{W}^{2}} \frac{h\left(\tilde{m}_{t 1}^{2}\right)-h\left(\tilde{m}_{t 2}^{2}\right)}{\tilde{m}_{t 1}^{2}-\tilde{m}_{t 2}^{2}}
$$

and the only difference is that $m_{A}$ is given by eq.(14).

\section{Results and Conclusion}

Resulting expressions for the Higgs masses differ from those obtained without RG summation. To calculate the corrections one has to perform the usual procedure [3], [4], [7] of fitting the set of soft breaking parameters, $m_{0}, m_{1 / 2}, \mu, \tan \beta, A$, as well as the top mass $m_{t}$. This is not a straightforward operation, since one has to fulfill many requirements simultaneously, thus defining the optimized best fit [7]. Having performed the complete procedure with the help of computer programm we have got the best fit values of the parameters mentioned above and used them in our formufae.

One of the important observation is the character of $m_{t}$ dependence of the results. Comparing with that obtained without account of the RG summation [7], we find it to be smoother. Fig.1 shows our results.

One should note, that $m_{t}$ in our formulae is the running top mass, which is connected with the physical (pole) top mass by the relation [9]:

$$
M_{t}^{\text {pole }}=m_{t}\left(1+\frac{4}{3} \frac{\alpha_{s}}{\pi}\right) \approx 1.06 m_{t}
$$

We conclude that the account of RG summation procedure can introduce the changes in the predictions of the Higgs masses. One can observe from Fig.1 that there exist the lower bound on the Higgs mass of about $95 \mathrm{GeV}$. In the interval of top mass preferable according to the recent $\mathrm{CDF}$ [10] data $\left(M_{t}^{\text {pole }}=174 \pm 10 \mathrm{GeV}\right)$, that corresponds to the running top mass of $164 \mathrm{GeV}$, the $m_{t}$ dependence of the lightest Higgs mass is very weak and $h$ appears to be lighter than $100 \mathrm{GeV}$.

\section{Acknowledgements}

The numerical analysis was performed with the help of the computer program, developed in [7]. We are grateful to W.de Boer and R.Ehret from Karlshrue University for valuable discussions and for necessary modification of the program. 


\section{References}

[1] J.Ellis, G.Ridolfi, F.Zwirner, Phys. Lett. B262 (1991) 477;

A.Brignole, J.Ellis, G.Ridolfi, F.Zwirner, Phys. Lett. B271 (1991) 123

[2] R.Arnowitt, P.Nath, Phys. Rev. D46 (1992) 3981

[3] G.G.Ross, R.G.Roberts, Nucl. Phys. B377 (1992) 571

[4] L.E.Ibáñez, C.Lopéz, Nucl. Phys. B233 (1984) 511;

L.E.Ibáñez, C.Lopéz, C.Muños, Nucl. Phys. B256 (1985) 218

[5] R.Barbieri, Riv. Nuo. Cim. 11 (1988) 1

[6] S.Coleman, E.Weinberg, Phys. Rev. D7 (1973) 1888

[7] W.de Boer, R.Ehret, D.I.Kazakov, IEKP-KA/94-05

[8] C.Ford, D.R.T.Jones, P.W.Stephenson, M.B.Einhorn, Nucl. Phys. B395 (1993) 17

[9] N.Gray, D.J.Broadhurst, W.Grafe, K.Schilscher, Z.Phys. C48 (1990) 673

[10] F.Abe et al., CDF Collab., FERMILAB-PUB-94/097-E 


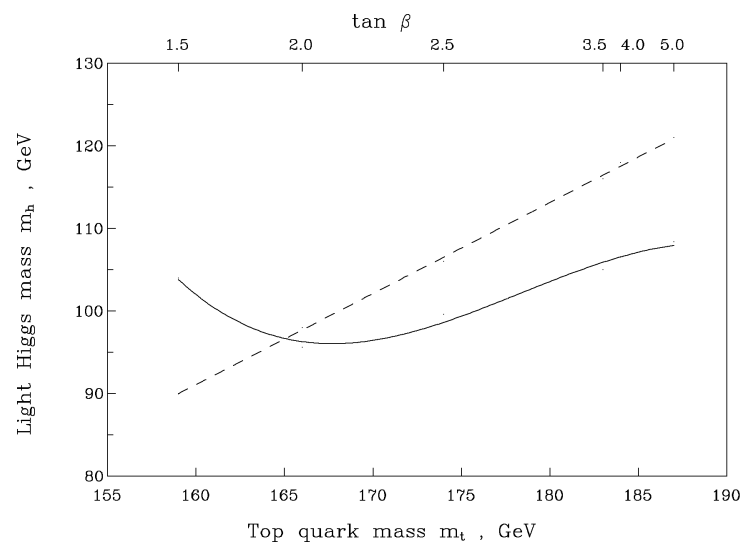

Figure 1: $m_{t}$ dependence of the light Higgs boson mass for $m_{0}=400 \mathrm{GeV}, m_{1 / 2}=$ $200 \mathrm{GeV}$. Dashed line corresponds to the 1-loop approximation, solid one corresponds to the $\mathrm{RG}$ improved radiative corrections. 
This figure "fig1-1.png" is available in "png" format from: http://arxiv.org/ps/hep-ph/9411209v1 